\title{
Pathogenesis of Polymicrobial Biofilms
}

\author{
Mary Ann Jabra-Rizk* \\ Department of Oncology and Diagnostic Sciences, University of Maryland, USA
}

\begin{abstract}
In polymicrobial biofilms a high level of interspecies interactions occur with often detrimental effect to the host. Many chronic infections are attributed to polymicrobial biofilms which tend to exhibit increased resistance to antimicrobial therapy. Yet despite the gravity of such infections, areas of study in polymicrobial diseases are in their infancy. Thus, much work is needed to promote a better understanding of emerging concepts in the biofilm development process such as interspecies communication and host immune response to microbial biofilms. The key challenges are to design effective therapeutic strategies to impede microbial colonization and prevent development of polymicrobial infections. Therefore, future research directions should focus on designing animal model systems to study in vivo-grown polymicrobial biofilms and infections. This review summarizes our limited knowledge about the nature of these complex communities and examines their role in disease, highlighting the challenges and novel approaches that are being pursued to combat polymicrobial biofilms and infections.
\end{abstract}

Keywords: Polymicrobial biofilms, interactions, Candida albicans, bacteria, infections.

\section{INTRODUCTION}

In most natural environments, microorganisms exist predominantly as multi species biofilms where intercellular interactions and communication are keys to survival [1-8]. Polymicrobial diseases, caused by combinations of viruses, bacteria, fungi, and parasites, are being recognized with increasing frequency [9]. The interactions between the various species in these mixed infections can be synergistic in that the presence of one microorganism generates a niche for other pathogenic microorganisms, predisposing the host to colonization or infection by a second organism [9]. In addition, the dense population structure in biofilms increases the opportunity of gene transfer between the species which can convert a previously avirulent commensal organism to a highly virulent pathogen [10]. This phenomenon of horizontal gene transfer is mediated mainly through bacterial plasmids, small, dispensable chromosomes which serve as vehicles that carry a considerable variety of genes. Some of these genes may be useful for the enhancement of survival under unfavorable conditions such as nutritional starvation and high cell density, two key characteristics of biofilm physiology [11-13]. Hence, plasmids including those that confer drug resistance and provide enzymes expand the nutritional ability of the cell and virulence determinants [13, 14]. The enhanced efficiency of gene transfer in biofilms induces enhanced stabilization of the biofilm structure but more importantly, also facilitates the spread of antibiotic resistance $[10,15]$. Therefore, understanding how antibiotic resistance develops and is spread by mobile genetic elements is a pre-requisite to the design of intervention strategies intended to minimize the threat of bacterial infections. Alternatively, the presence of one microorganism may

*Address correspondence to this author at the Department of Oncology and Diagnostic Sciences, Dental School, University of Maryland, $650 \mathrm{~W}$ Baltimore Street, 7 N, Room 2753, Baltimore, MD 21201, USA; Tel: (410)708-0508; Fax: (410)-706-0519; E-mail: mrizk@umaryland.edu generate a niche in the host that suppresses the colonization of other microorganisms. This form of interaction is called "microbial interference" [9]. This review outlines our understanding of the nature of polymicrobial interactions in biofilm within the context of human disease with emphasis on novel prophylactic and therapeutic strategies targeting polymicrobial biofilms.

\section{THE ART OF COMMUNICATION}

In a biofilm environment, microbial species are highly interactive and employ a range of cell-to-cell communication or 'quorum sensing' systems [16-18]. This phenomenon for promoting collective behavior with in a population is important in ensuring survival and propagation by enhancing access to nutrients and niches, as well as providing protection $[4,5,19]$. Although much of what we understand today came from the study of single species biofilm, it is now clear that in the natural and clinical environments, most biofilms are likely to consist of consortia of species that influence each other in synergistic and antagonistic manners $[4,8,20]$. However, limited studies have specifically addressed interactions within multi species biofilms and particularly interactions between bacteria and fungi, which are often found together in a myriad of environments [16, 21-23]. Although the area of research exploring interkingdom interactions in biofilm is still in its infancy, there is increasing awareness of their clinical implications in the host particularly between the fungal pathogen Candida albicans and various bacterial species (for detailed description of some of these interactions within the context of human disease, the reader is referred to two recent reviews) [22, 23].

C. albicans is the major fungal pathogen of humans causing a variety of afflictions ranging from superficial mucosal diseases to deep seated mycoses [24-26]. Biofilm formation is a major virulence factor in the pathogenicity of C. albicans and Candida biofilms are difficult to eradicate 
due to their high resistance to antifungals [24, 26, 27]. Consequently, research into the pathogenicity of C. albicans has focused on the prevention of biofilm development and management of drug resistance [28]. A recent study examining the structure of biofilm formed by $C$. albicans and the bacterial pathogen Staphylococcus aureus as they coexist revealed a unique architecture where $S$. aureus associated with the hyphae of $C$. albicans. Further characterization of this seemingly synergistic type of interaction at the molecular level demonstrated significant level of differential protein expression the result of the mixed-species biofilm mode of growth [29]. Interestingly, a number of these proteins were identified to be virulence factors in S. aureus indicating a process whereby C. albicans may enhance $S$. aureus pathogenesis [29]. These findings are of great significance as these two species are currently ranked as the second and third most commonly isolated bloodstream pathogens in hospitalized patients [24].

Another form of commensalism described in multispecies biofilms is "indirect pathogenicity" which was recognized during treatment failure of polymicrobial infections. This phenomenon describes an interactive association where one organism benefits while the other is not affected [30]. For example, in a mixed infection, an antibiotic-resistant microorganism of low intrinsic virulence protects an antibiotic-sensitive pathogen from eradication $[30,31]$. Interestingly, a similar phenomenon was recently described for $S$. aureus and $C$. albicans where the coexistence of these human pathogens in a biofilm resulted in increased $S$. aureus resistance to vancomycin [32]. Alternatively, limited space and nutrients in biofilms can lead to competition between microorganisms resulting in antagonistic interactions, typified by one organism's direct, deleterious impact on another. An example of such antagonistic interaction is the one described between Pseudomonas and Agrobacterium, in which growth rate and motility impacted the fitness of each competitor [33]. However, a more clinically relevant antagonistic interaction is the one reported between $P$. aeruginosa and C. albicans where $P$. aeruginosa was shown to form dense biofilm on $C$. albicans hyphae and kill the fungus [34-36](Fig. (1) depicts the adherence of $S$. aureus and $P$. aeruginosa to $C$. albicans hyphae in biofilm).

\section{THE ORAL MICROBIOME}

The human mouth with its diverse niches and ample supply of nutrients is undoubtedly conducive for the unrestricted formation of natural microbial biofilms, such as those found on the tooth as dental plaque and oral mucosal tissue where a multitude of microbial species co-exist [31, 37]. Recent technological advances in metagenomic analyses have enhanced the identification and characterization of the vast microbial diversity colonizing the human body. The oral microbial communities are some of the most complex microbial floras in the human body, consisting of more than 700 different bacterial species $[31,38]$. The analysis of the diversity and distributions of microorganisms in oral biofilm communities through microbiome studies, has allowed insight into the differences between the normal state of the oral microbiota and the alterations that are present during disease states $[31,38]$.

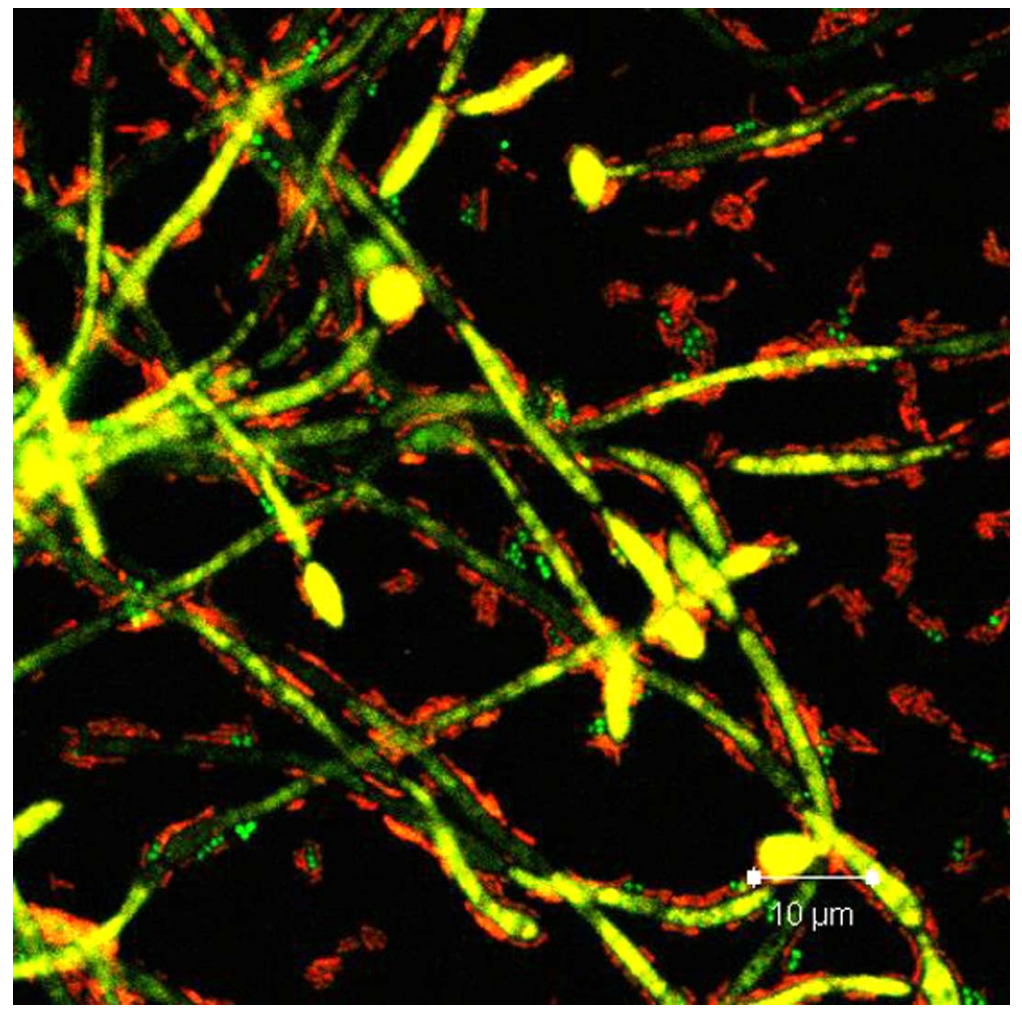

Fig. (1). Fluorescence in situ hybridization image of C. albicans and S. aureus mixed-species biofilm using fluoresce in and Tamra-labeled species-specific peptide nucleic acid probes demonstrating extensive adherence of $S$. aureus (green) and $P$. aeruginosa (red) to C. albicans hyphae (yellow). 
In the oral cavity, $C$. albicans co-exist and form tight associations with various oral bacterial species. The range of intergeneric coaggregations occurring between $C$. albicans and oral species likely plays an important factor in $C$. albicans colonization where bacteria modulate fungal growth and biofilm formation [31]. Streptococcus gordonii specifically which is found on most oral cavity surfaces have been shown to interact with $C$. albicans to promote hyphaland biofilm-formation [39]. The C. albicans $A L S$ genes encoding a family of adhesins have been implicated in the adherence of $C$. albicans to surfaces including host tissue and bacteria. $A L S 3$ specifically, which encodes a hyphal cell wall-specific protein (Als3p) with adhesive properties was recently shown to be involved in the interactions of $C$. albicans with $S$. gordonii. Recently, a coaggregation study examining the interaction between, $S$. gordonii cells and $C$. albicans demonstrated that although the streptococci attached to the hyphae formed by C. albicans wild-type cells, they failed to attach to the hyphae produced by an $A L S 3$ deletion mutant strain [39]. In another study, the adhesive role of the Als3p was further demonstrated by blocking adhesion of C. albicans to buccal epithelial cells with immunoglobulin reactive against the Als3p N-terminal sequences [40].

The interactions between $C$. albicans and streptococci specifically, appear to be essentially synergistic where in addition to providing adhesion sites, the streptococci excrete lactate that can act as a carbon source for yeast growth [31, 41, 42]. More importantly, the most serious ramification of these fungal-bacterial interactions with clinical implications comes from findings demonstrating that the physical interactions between $C$. albicans and oral streptococci increased tolerance of the polymicrobial biofilm to antimicrobial agents and enhanced resilience to physical disruption [31]. Thus, when Candida infections arise, they often occur in association with bacteria. On the other hand, there is also strong evidence to suggest that components of the resident microflora present in the oral cavity and at other mucosal sites, perform to check C. albicans growth. This is why factors that perturb the normal microflora, such as antibiotic therapy or changes in hormonal or mucosal secretions, may encourage C. albicans overgrowth. A study evaluating the effect of eight aerobic and anaerobic oral commensal bacterial species on the growth and survival of C. albicans biofilms indicated that the quantitative and qualitative nature of the bacteria, modulate C. albicans biofilm formation in the oral cavity [43]. Similarly, a more recent study characterizing oral mucosal C. albicans biofilms concluded that $C$. albicans forms complex mucosal biofilms consisting of both commensal bacterial flora and host components [44]. Therefore, understanding the complex mechanisms by which Candida and oral bacteria co-colonize will assist in the development of new protocols to block adhesive reactions and eliminate Candida from biofilmrelated oral infections such as denture stomatitis.

\section{NOVEL STRATEGIES TO COMBAT POLYMICRO- BIAL BIOFILMS AND INFECTIONS}

Many nosocomial infections involve microbial biofilms and persistence of chronic infections is attributed to the persistence of polymicrobial biofilms $[9,45]$. In these situations, traditional therapies are generally targeted at individual causative agents without consideration for effect on a polymicrobial cause or on individual members of microbial communities. The standard treatment regimen employed for polymicrobial infections involves two or more antibiotics, referred to as combination therapies $[9,46]$. The use of novel antibiotic combinations and antibiotic cycling may prolong the effectiveness of antibiotic therapies [47]. However, a careful attempt should be made to identify the causative microorganisms, as appropriate management of mixed infections requires the administration of antimicrobials that are effective against both components of the infection [46]. Significantly, the increasing emergence of drug resistance to commonly used antibiotics and antifungals has made the need for the identification of novel therapeutics and approaches critical. Therefore, development of effective strategies to control or prevent biofilm-associated infections requires a thorough understanding of the biofilm development process [48].

The medical community is recognizing the significance of polymicrobial diseases and the major types of microbial community interactions associated with human health and disease. Therefore, design of novel therapeutic strategies is just starting to take into account the polymicrobial cause of diseases and the repercussions on treatment and prevention [9]. Among the promising approaches to combat biofilm infections is the generation of surface modification of devices to reduce microbial attachment and biofilm development, as well as incorporation of antimicrobial agents to prevent colonization [49]. Similarly, several compounds and synthetic analogues have been used successfully to prevent biofilm formation such as farnesol, which was shown to effectively inhibit bacterial and fungal biofilm formation $[50,51]$. Another option could be to coat biomaterial surfaces with organic molecules to prevent protein adsorption which may also inhibit biofilm formation [52]. Further, the biofilm matrix is composed of a variety of structural components, including DNA in addition to polysaccharides and proteins. Therefore, a promising strategy is the use of substances and enzymes (e. g. DNase and alginate lyase) able to disrupt and dissolve biofilms by attacking surface polysaccharides and the extracellular DNA which is critical for the early development of biofilms [53]. Along those lines, other innovative approaches consist of disrupting biofilms by exposing them to photodynamic substances [52]. Typically, biofilms must release and disperse cells into the environment in order to colonize new sites [54]. Therefore, biofilm dispersal is another promising area of research that may lead to the development of novel agents to promote biofilm cell detachment.

A new mechanism for novel prophylactic or therapeutic management of polymicrobial diseases targets another biofilm property, microbial interference, through the use of probiotics. The use of antibiotics and immunosuppressives often causes alterations in the composition of host microflora particularly in the oral cavity and the intestinal and urogenital tracts. Therefore, the introduction of beneficial microbial species may be a very attractive option to reestablish the microbial equilibrium and prevent disease [55]. Among the bacterial genera used in probiotic preparations are Lactobacillus, Bifidobacterium, Escherichia, Enterococcus, Bacillus and Streptococcus in addition to the fungal 
species belonging to Saccharomyces. Through immune modulation pathogen displacement and creation of a niche less conducive to proliferation of pathogens and their virulence factors, probiotics were shown to be effective in varied clinical conditions such as antibiotic-associated diarrhea and Helicobacter pylori infections [55-57]. One good example is using lactobacilli to improve urogenital health in women.

Alternatively, the realization that a number of pathogens utilize the process of quorum sensing to establish a biofilm and control much of their virulence arsenal by means of extracellular signal molecules has identified the communication machinery as new drug targets. In fact, the process of quorum sensing was shown to be involved in the development of resistance to various antimicrobial treatments and immune modulation. Therefore, the use of quorum-sensing inhibitors that block communication may control biofilm formation, increase biofilm susceptibility to antibiotics as well as the susceptibility of the pathogens to host defenses [17, 58, 59]. Although quorum sensing antagonists hold great promise in fighting polymicrobial biofilms they should be viewed as blockers of pathogenicity rather than as antimicrobials. However, to define potential new strategies for impeding microbial colonization and development of polymicrobial disease will require a thorough understanding of the mechanisms of adhesion and inter-cellular signaling involved in mixed-species interactions.

Normal mucosal surfaces resist biofilm infections despite continual exposure to commensal and pathogenic microorganisms indicating that mucosal surfaces might possess an anti-biofilm defense. Yet, although much work has been done to address the role of biofilm mode of growth in antimicrobial resistance there have been very few studies addressing the role of biofilm resistance to the human immune system or the host response to polymicrobial biofilms. Therefore, much work is needed to promote a better understanding of host response, both innate and adaptive to microbial biofilms and future research should be directed towards studying the immunology of biofilms. Development of high throughput methods to identify host immunologic factors that are differentially expressed in biofilms may lead to the design of processes for enhancement of the role of innate immune factors in prevention or elimination of biofilms.

\section{CONCLUSION AND FUTURE PERSPECTIVES}

Although it is important to continue studies of the pathogenic properties of specific microbes, understanding the microbial communities and their interactions that drive sickness or health is key to combating polymicrobial diseases [31]. Yet despite their seriousness, expansive research into the area of polymicrobial infections have been lacking. A deeper understanding of the interactions involved in polymicrobial biofilms will provide a new perspective on the factors relevant to polymicrobial disease. The key challenges no ware to design strategies to prevent development of polymicrobial infections using emerging concepts such as interspecies interaction. To that end, new research directions should focus on designing animal model systems to study in vivo-grown polymicrobial biofilms and infections.

\section{REFERENCES}

[1] Douglas LJ. Medical importance of biofilms in Candida infections. Rev Iberoam Micol 2002; 19: 139-43.

[2] Lewis K. Riddle of biofilm resistance. Antimicrob Agents Chemother 2001; 45(4): 999-1007.

[3] Lynch AS, Robertson GT. Bacterial and fungal biofilm infections. Ann Rev Med 2008; 59: 415-28.

[4] Moons P, Michiels CW, Aertsen A. Bacterial interactions in biofilms. Crit Rev Microbiol 2009; 35(3): 157-68.

[5] Nadell CD, Xavier JB, Foster KR. The sociobiology of biofilms. FEMS Microbiol Rev 009; 33(1): 206-24.

[6] Wargo MJ, Hogan DA. Fungal-bacterial interactions: a mixed bag of mingling microbes. Curr Opin Microbiol 2006; 9: 359-64.

[7] Donlan RM, Costerton JW. Biofilms: survival mechanisms of clinically relevant microorganisms. Clin Microbiol Rev 2002; 15(2): 167-93.

[8] Stoodley P, Sauer K, Davies DG, Costerton JW. Biofilms as complex differentiated communities. Ann Rev Microbiol 2002; 56(1): 187-209.

[9] Brogden KA, Gerberding JM, editors. Polymicrobial Diseases. Washington: ASM Press 2002.

[10] Molin S, Tolker-Nielson. T Gene transfer occurs with enhanced efficiency in biofilms and induces enhanced stabilisation of the biofilm structure. Curr Opin Biotechnol 2003; 14(3): 255-61.

[11] Elsas JD BM. The ecology of transfer of mobile genetic elements. FEMS Microbiol Ecol 2002; 42(2): 187-97.

[12] Fux CA, Costerton JW, Stewart PS, Stoodley P. Survival strategies of infectious biofilms. Trends Microbiol 2005; 13(1): 34-40.

[13] Bennett P. Plasmid encoded antibiotic resistance: acquisition and transfer of antibiotic resistance genes in bacteria. Br J Pharmacol 2008; 153 (S1): 347-57

[14] Hogan D, Kolter R. Why are bacteria refractory to antimicrobials. Curr Opin Microbiol 2002; 5(5): 472-7.

[15] Wuertz SOS. Hausner M. Microbial communities and their interactions in biofilm systems: an overview. Water Sci Technol 2004; 49(11,12): 327-36.

[16] Hogan DA. Talking to themselves: autoregulation and quorum sensing in fungi. Eukaryot Cell 2006; 5(4): 613-9.

[17] Jayaraman A, Wood TK. Bacterial Quorum sensing: signals, circuits, and implications for biofilms and disease. Ann Rev Biomed Engin 2008; 10(1): 145-67.

[18] Williams P. Quorum sensing, communication and cross-kingdom signalling in the bacterial world. Microbiol 2007; 153: 3923-38.

[19] Nikolaev YA, Plankunov VK. Biofilm- "city of microbes" or an analogue of multicellular organisms? Microbiology 2007; 76(2): 125-38.

[20] Watnick P, Kolter R. Biofilm, city of microbes. J Bacteriol 2000; 182(10): 2675-9

[21] Joint I, Tait K, Callow ME, et al. Cell-to-cell communication across the prokaryote-eukaryote boundary. Science 2002; 298(5596): 1207.

[22] Morales DK, Hogan DA. Candida albicans interactions with bacteria in the context of human health and disease. PLoS Path6(4): e1000886.

[23] Shirtliff ME, Peters BM, Jabra-Rizk MA. Cross-kingdom interactions: Candida albicans and bacteria. FEMS Microbiol Lett 2009; June: 1-8.

[24] Perlroth J, Choi B, Spellberg B. Nosocomial fungal infections: epidemiology, diagnosis, and treatment. Med Mycol 2007; 45(4): 321-46.

[25] Seneviratne C, Jin L, Samaranayake L. Biofilm lifestyle of Candida: a mini review. Oral Dis 2008; 14(7): 582-90.

[26] Seneviratne G, Zavahir JS, Bandara WMMS, Weerasekara MLMAW. Fungal-bacterial biofilms: their development for novel biotechnological applications. World J Microbiol Biotech 2008; 24: 739-43.

[27] Mukherjee PK, Zhou G, Munyon R, Ghannoum MA. Candida biofilm: a well-designed protected environment. Med Mycol 2005; 43: 191-208.

[28] Mukherjee PK, Chandra J. Candida biofilm resistance. Drug Resist Updates 2004; 7(4,5): 301-9.

[29] Peters BM, Jabra-Rizk MA, Scheper MA, et al. Microbial interactions and differential protein expression in Staphylococcus aureus and Candida albicans dual-species biofilms. FEMS Imm Med Microbiol 2010; 59: 493-503. 
[30] O'Connell HA, Kottkamp GS, Eppelbaum JL, Stubblefield BA, Gilbert SE, Gilbert ES. Influences of biofilm structure and antibiotic resistance mechanisms on indirect pathogenicity in a model polymicrobial biofilm. Appl Environ Microbiol 2006 2006; 72(7): 5013-9.

[31] Jenkinson HF, Lamont RJ. Oral microbial communities in sickness and in health. Trends Microbiol 2005; 13(12): 589-95.

[32] Harriott MM, Noverr MC. Candida albicans and Staphylococcus aureus form polymicrobial biofilms: effects on antimicrobial resistance. Antimicrob Agents Chemother 2009; 53(9): 3914-22.

[33] An D, Danhorn T, Fuqua C, Parsek MR. Quorum sensing and motility mediate interactions between Pseudomonas aeruginosa and Agrobacterium tumefaciens in biofilm cocultures. Proc Natl Acad Sci USA 2006; 103(10): 3828-33.

[34] McAlester G, O'Gara F, Morrissey JP. Signal-mediated interactions between Pseudomonas aeruginosa and Candida albicans. JMed Microbiol 2008; 57: 563-9.

[35] Hogan DA, Kolter R. Pseudomonas-Candida interactions: an ecological role for virulence factors. Science 2002; 296(5576): 2229-32.

[36] Ramage G, Saville SP, Thomas DP, Lopez-Ribot JL. Candida biofilms: an update. Eukaryot Cell 2005; 4(4): 633-8.

[37] Nasidze I, Quinque D, Li J, Li M, Tang K, Stoneking M. Comparative analysis of human saliva microbiome diversity by barcoded pyrosequencing and cloning approaches. Analyt Biochem 2009; 391(1): 64-8.

[38] Dewhirst Fe, Chen T, Izard J, et al. The human oral microbiome. J Bacteriol 2010; JB. 00542-10

[39] Silverman RJ, Nobbs AH, Vickerman MM, Barbour ME, Jenkinson HF. Interaction of Candida albicanscell-wall Als3 protein with Streptococcus gordonii $\mathrm{SspB}$ adhesin promotes development of mixed species communities. Infect Immun 2010: IAI. 00685-10.

[40] Zhao X, Daniels KJ, Oh S-H, et al. Candida albicans Als3p is required for wild-type biofilm formation on silicone elastomer surfaces. Microbiology 2006; 152(8): 2287-99.

[41] Holmes AR, Cannon RD, Jenkinson HF. Interactions of Candida albicans with bacteria and salivary molecules in oral biofilms. JInd Microbiol 1995; 15(3): 208-13.

[42] Jenkinson HF, Lala HC, Shepherd MG. Coaggregation of Streptococcus sanguis and other streptococci with Candida albicans. Infect Immun 1990; 58(1): 1429-36.

[43] Thein ZM, Samaranayake YH, Samaranayake L. Effect of oral bacteria on growth and survival of Candida albicans biofilms. ArchOral Biol 2006; 51(8): 672-80.
[44] Dongari-Bagtzoglou A, Kashleva H, Dwivedi P, Diaz P, Vasilakos J. Characterization of mucosal Candida albicansbiofilms. PLoS ONE 2009; 4(11): e7967.

[45] Hall-Stoodley L, Stoodley P. Evolving concepts in biofilm infections. Cell Microbiol 2009; 11(7): 1034-43.

[46] Brook I. Microbiology of polymicrobial abscesses and implications for therapy. J Antimicrob Chemother 2002; 50(6): 805-10.

[47] Masterton RG. Antibiotic cycling: more than it might seem. J Antimicrob Chemother 2005; 55(1): 1-5.

[48] Jain A GY, Agrawal R, Khare P, Jain SK. Biofilms a microbial life perspective: a critical review. Crit Rev Ther Drug Carrier Syst 2007; 24(5): 393-443.

[49] Smith AW. Biofilms and antibiotic therapy: is there a role for combating bacterial resistance by the use of novel drug delivery systems. Adv Drug Deliv Rev 2005 Jul; 57(10): 1539-50.

[50] Shirtliff ME, Krom BP, Meijering RA, et al. Farnesol-induced apoptosis in Candida albicans. Antimicrob Agents Chemother 2009; 53(6): 2392-401.

[51] Jabra-Rizk MA, Meiller TF, James C, Shirtliff ME. Effect of farnesol on Staphylococcus aureus biofilm formation and antimicrobial resistance. Antimicrob Agents Chemother 2006; 50(4): 1463-9.

[52] Njoroge J, Sperandio V. Jamming bacterial communication: New approaches for the treatment of infectious diseases. EMBO Mol Med 2009; 1(4): 201-10.

[53] Arciola C. New concepts and new weapons in implant infections. Int J Artif Organs 2009; 32(9): 533-6.

[54] Kaplan JB. Biofilm dispersal: mechanisms, clinical implications and potential therapeutic uses. J Dent Res 2010; 89(3): 205-18.

[55] Gupta V GR. ProbioticsIndian J Med Microbiol 2009; 27(3): 2029.

[56] Dobrogosz WJ, Peacock TJ, Hassan HM, Allen I. Laskin SS, Geoffrey MG. Evolution of the probiotic concept: from conception to validation and acceptance in medical science. Adv Appl Microbiol 2010; 72: 1-41.

[57] Reid G, Tieszer C, Lam D. Influence of lactobacilli on the adhesion of Staphylococcus aureus and Candida albicans to fibers and epithelial cells. J Ind Microbiol 1995; 15(3): 248-53.

[58] Hoiby N, Bjarnsholt T, Givskov M, Molin S, Ciofu O. Antibiotic resistance of bacterial biofilms. Int J Antimicrob Agents 2010; 35(4): 322-32.

[59] Bjarnsholt T, Givskov M. Quorum-sensing blockade as a strategy for enhancing host defences against bacterial pathogens. Philosophical Transactions of the Royal Society B. Biol Sci 2007; 362(1483): 1213-22. 